\title{
In situ visualization of superior nanomechanical flexibility of individual ydroxyapatite nanobelts
}

Mei-li Qi ${ }^{1}$, Zhen-nan Huang ${ }^{2}$, Wen-tao Yao ${ }^{3}$, Fei Long ${ }^{4}$, Meng Cheng ${ }^{5}$, Boao Song ${ }^{6}$, David Banner ${ }^{5}$, Reza Shahbazian-Yassar ${ }^{7}$, Yu-peng $\mathrm{Lu}^{8}$ and Tolou Shokuhfar ${ }^{5}$

${ }^{1}$ Shandong Jiaotong University, Ji'nan, China (People's Republic), ${ }^{2}$ University of Illinois at Chicago, Chicago, United States, ${ }^{3}$ Michigan Technological University, United States, ${ }^{4}$ Michigan Technological University, Houghton, Michigan, United States, ${ }^{5}$ University of Illinois at Chicago, United States, ${ }^{6}$ Eurofins, United States, ${ }^{7}$ University of Illinois at Chicago, Chicago, Illinois, United States, ${ }^{8}$ Shandong University, United States

The biocompatibility, bioactivity, osteoconductivity and nontoxic properties of synthetic hydroxyapatite (HA) with controlled sizes and morphologies are ideal in bone repair, tissue engineering, drug delivery, and related applications. However, the brittleness and poor fatigue resistance of synthetic HA greatly limits its applications for load-bearing materials. Molten salt, hydrothermal, sol-gel, electrospinning and electroblowing methods were developed to synthesize flexible HA structures. However, synthesizing HA nanostructures with uniform morphology and high aspect ratio $(>100)$ is still an experimental challenge. Measuring the flexibility of synthesized HA and the corresponding flexion mechanisms present additional technical challenges. Flexibility has been mainly described through bent morphology, static microstructures, or macroscopic tensile testing. These methods, however, cannot provide direct observation of the microscopic structure alternations in real-time and only infer the flexibility mechanism. It is of crucial importance to identify whether the HA structures with bending phenomenon is flexible.

Recently, in situ transmission electron microscopy (TEM) has been used as an effective tool for direct measurement of an individual structure such as $\mathrm{TiO} 2$ nanotubes, shear-exfoliated phosphorene nanowires and graphene ribbons. This electron microscopy (EM) approach measures the properties of individual structures and records structural changes during loading through advanced electron imaging and diffraction techniques, providing an ideal experimental setup for the dynamic study of nanoscale propertystructure relationships.

In this paper, we synthesized highly flexible hydroxyapatite (HA) nanobelts with uniform morphology and high aspect ratios by a one-step hydrothermal homogeneous precipitation method. More importantly, we for the first time, directly visualized the nanomechanical behaviour of individual HA nanobelts by an in situ nano-manipulation system under TEM. The nanobelts can be easily bent, scrolled, and stretched through seven times of cyclic compression tests, showing remarkable nanomechanical flexibility rather than fracturing. Further, nanoindentation tests were conducted, indicating that the HA nanobelt has a better resistance to deformation as well as a more reduced modulus, which is consistent with the in situ TEM tests. The results support the application of HA nanobelts as load-bearing implants, reinforcements in biomedical composite materials, and scaffolds for the tissue engineering. 

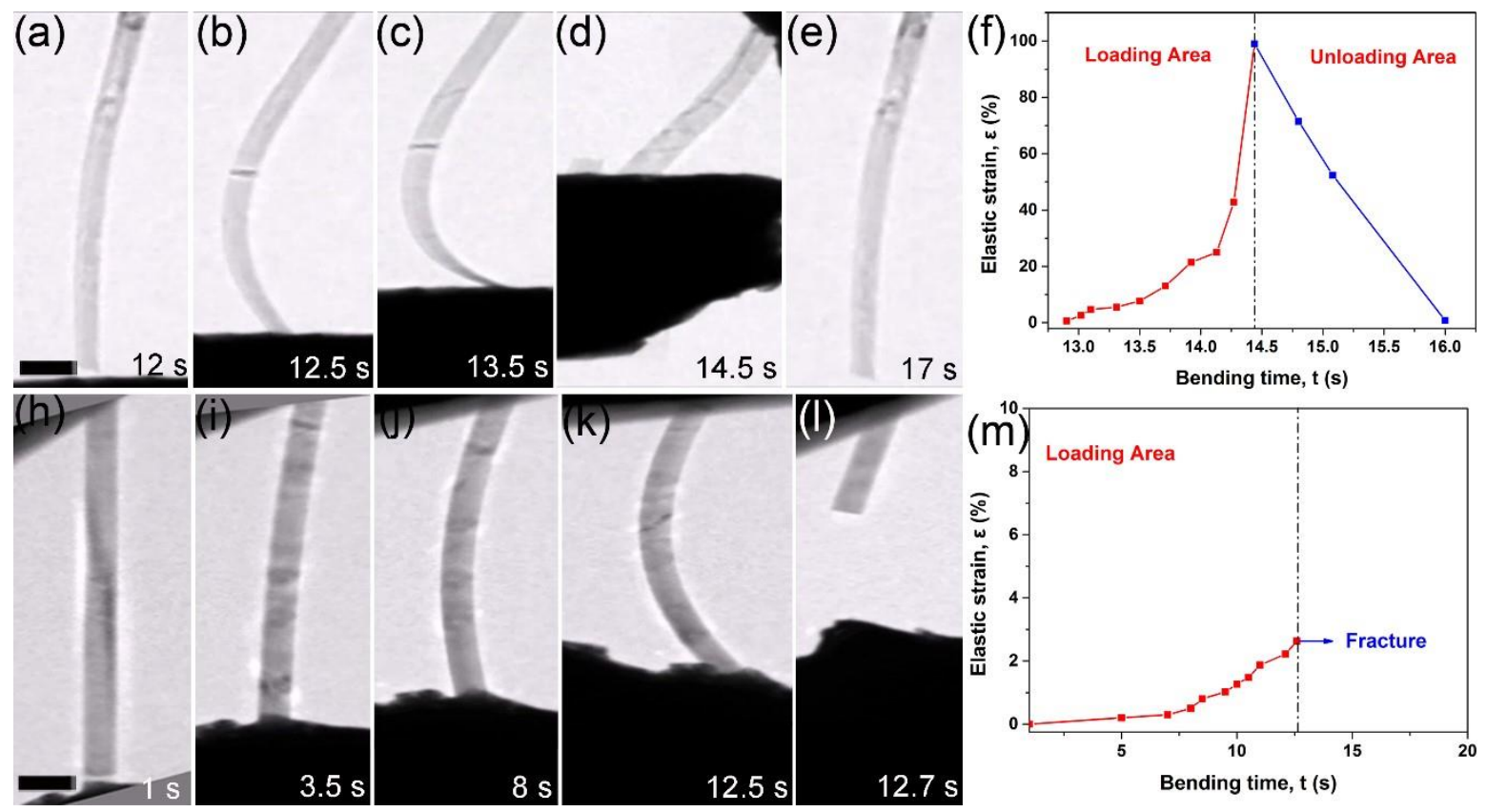

Figure 1. (a-e) Sequential morphological evolution of the HA nanobelt under the seventh severe compression cycle, and (f) the corresponding $\varepsilon$-t curve of the nanobelt during this process, (h-1) Sequential morphological of the HA nanorod during compression, and (m) the corresponding $\varepsilon-\mathrm{t}$ curve of the nanorod during this process. Scale bar is $200 \mathrm{~nm}$.

\section{References}

1. Qi M, Huang Z, Yao W, et al. In situ visualization of the superior nanomechanical flexibility of individual hydroxyapatite nanobelts[J]. CrystEngComm, 2018, 20(8): 1031-1036.

2. Xu F, Ma H, Lei S, et al. In situ TEM visualization of superior nanomechanical flexibility of shearexfoliated phosphorene[J]. Nanoscale, 2016, 8(28): 13603-13610.

3. Espinosa H D, Bernal R A, Filleter T. In situ TEM electromechanical testing of nanowires and nanotubes[J]. Small, 2012, 8(21): 3233-3252.

4. This work is financially supported by the National Natural Science Foundation of China (11947117, 12004227 and 51803109), the Natural Science Foundation of Shandong Province (ZR2020QE070 and ZR2020QA076) and China Postdoctoral Science Foundation (2019M660164). 